\title{
BASES PARA PRESCRIÇÃO DO TREINAMENTO DESPORTIVO APLICADO AO BRAZILIAN JIU-JITSU
}

Leonardo Vidal Andreato

\section{Resumo}

Nos dias atuais o condicionamento físico vem se tornando cada vez mais importante, tornando-se fator determinante para se alcançar o triunfo nos esportes que exigem das aptidões físicas, em alguns casos superando o talento. Contudo, a sistematização da preparação física deve ser realizada respeitando teorias gerais do treinamento desportivo e principalmente as especificidades da modalidade. Neste sentido, o Brazilian Jiu-Jitsu é um esporte que vem crescendo muito nos últimos anos, principalmente em seu caráter esportivo, porém a evolução literária acerca da modalidade não segue a mesma proporção. Desta forma, o objetivo deste estudo foi por meio bibliográfico, explanar algumas das bases para a prescrição do treinamento desportivo aplicado ao Brazilian Jiu-jitsu.

\section{Palavras-Chave}

Treinamento desportivo; Alto rendimento; Brazilian jiu-jitsu.

\section{BASES FOR PRESCRIPTION IN SPORTS TRAINING APLICATEDED TO JIU-JITSU}

Leonardo Vidal Andreato

\begin{abstract}
Nowadays physical fitness is becoming each more important, it's becoming a determinant factor to be well succeed in sports that require physical capacities and in some cases surpassing talent. However, physical preparation's systematization must be implemented within general theories of sports training and especially the sport's specifics. In this sense, Brazilian Jiu-Jitsu is a sport that has rising in a significative way lately especially in sportive character, but literature development abut the sport does not follow the same proportion. Thus, this study was by literature, it intended to explain some bases for sports training prescription applied to Brazilian jiu-jitsu.
\end{abstract}

\section{Key-Words}

Sports training; High performance; Brazilian jiu-jitsu. 


\section{INTRODUÇÃO}

Nos dias atuais o condicionamento físico vem se tornando cada vez mais importante, tornando-se fator determinante para se alcançar o triunfo nos esportes que exigem das aptidões físicas, em alguns casos superando o talento. Desta forma inúmeros atletas, técnicos e preparadores desprendem cada vez mais importância para a preparação física (FORTEZA, 1999; GOMES, 2001). Esportes competitivos, como o Brazilian Jiu-Jitsu, exigem treinamentos intensos, havendo sem dúvida sobrecarga ao corpo humano. Nos esportes de contato esta sobrecarga é ainda maior, pois ainda está envolvido o peso do outro atleta (ASSIS; GOMES; CARVALHO, 2005).

Cabe ao preparador físico criar condições ao atleta onde se possa atingir o rendimento e preservar a saúde (GOMES, 1999). Este por sua vez é identificado como o fator mais importante no processo de desenvolvimento das habilidades dos atletas, principalmente em aspectos de aprendizagem, refinamento técnico e aquisição de estratégias para a competição (LOBO; MORAES; NASCIMENTO, 2005).

$\mathrm{O}$ treinamento físico pode ser entendido como o conjunto de meios utilizados para o desenvolvimento das qualidades técnicas, físicas e psicológicas de um atleta ou de uma equipe, tendo como objetivo final obter o melhor desempenho na fase certa (TUBINO, 1984). Entretanto, o treinamento físico é um conceito muito complexo, o qual abrange conhecimentos nas áreas de aprendizagem motora, pedagogia do esporte, medicina do esporte, biomecânica, bioquímica, fisiologia do exercício, anatomia, psicologia do esporte, e sociologia do esporte. $\mathrm{O}$ mesmo dever ser planejado, organizado e conduzido por pessoas especialistas e competentes (BARBANTI, 2001).

O Brazilian Jiu-Jitsu engloba diversas capacidades e aptidões como força, flexibilidade, velocidade, agilidade, potência aeróbia e anaeróbia. Em seu modelo competitivo, possui diversas divisões de atletas em categorias, respeitando idade, peso, graduação e sexo. No entanto, existe pouco acervo literário acerca do Brazilian Jiu-Jitsu, fato este que dificulta o entendimento e planejamento do treinamento da modalidade. Devido a esta dificuldade, dados referente a modalidades semelhantes como Judô e Luta olímpica são utilizados.

Desta forma, o presente estudo tem como objetivo fornecer dados que contribuam para o entendimento do Brazilian Jiu-Jitsu, e que possam ser utilizados no planejamento do treinamento desportivo. 


\section{SISTEMAS ENERGETICOS APLICADOS AO JIU-JITSU}

A principal característica fisiológica de lutas como o Judô é a intermitência, que seria uma série de esforços supra-máximos intercalados por alguns instantes de recuperação, onde são realizadas atividades de pequena intensidade ou repouso. No judô têm se a proporção de 15 a 30 segundos de luta por 10 a 15 segundos de intervalo. Sendo considerado uma modalidade com predominância do metabolismo anaeróbio láctico (ARTIOLI et al., 2006; FRANCHINI et al., 1999). Quanto à proporção luta/pausa no Brazilian Jiu-Jitsu, em análise de 33 lutas da Copa do Mundo de 2005, obtive-se a proporção de tempo médio de luta em pé de 25,4 segundos, luta em solo de 145,7 segundos, e tempo de recuperação de 13,06 segundos. Somando os componentes de luta em pé e luta no solo, atinge-se uma proporção de 170 segundos de luta por 13 segundos de recuperação (DEL VECCHIO et al., 2007).

Nesses curtos intervalos, não há tempo suficiente para a ressíntese de ATP pelas vias aeróbias, o que torna os esforços dependentes da via anaeróbia láctica. A importância da via glicolítica pode ser expressa pela elevada concentração de lactato sangüíneo [LAC] (ARTIOLI et al., 2006). Atletas de Jiu-Jitsu (n=7) apresentaram valores de [LACpré] de 2,3 \pm 0,2 $\mathrm{mMol}$ e 2 minutos após uma luta [LAC pós 2$]_{10,2} \pm 2,4$ mMol, denotando a predominância da via anaeróbia láctica (DEL VECHIO et al., 2007). Em atletas de Judô ( $\mathrm{n}=8$ ) não foi apresentada diferenças significativas da [LAC] da luta de solo e luta em pé (DRIGO et al., 1996). Lima e colaboradores (2004) em estudo com judocas $(n=11)$ para analisar a influencia da [LAC] sobre a velocidade de reação concluíram que as altas [LAC] fazem com que ocorra uma diminuição na eficiência da tarefa de tempo de reação.

\section{FREQUÊNCIA CARDÍACA APLICADA AO JIU-JITSU}

A freqüência cardíaca (FC) pode ser um indicativo da intensidade do esporte e indicativo da predominância de sistema fornecedor de energia (POWERS; HOWLEY, 2000; WILMORE; COSTIL, 2001). Em estudo com lutadores de BJJ ( $n=7)$ onde estes realizaram lutas entre 7 e 10 minutos, obtive-se os seguintes valores: a FC pré-luta de 72,2 \pm 9,2 batimentos por minuto (bpm), e FC média de 181,7 $\pm 5,9$ bpm; FC máxima ( $\mathrm{FC}_{\text {máx }}$ ) de 195,0 \pm 7,1 bpm. Concluí-se com este estudo que a FC tende aumentar de forma não linear (DEL VECCHIO et al., 2007). Franchini et al. (2003) em estudo com praticantes de Brazilian Jiu-Jitsu ( $\mathrm{n}=22)$ relatam quem em uma luta de 5 minutos a maior FC é apresentada no último minuto, embora o seu aumento não ocorra de forma linear.

$\mathrm{O}$ aumento da FC de forma não linear pode estar relacionado ao fato que ocorre predominância de pontuação até o terceiro minuto da luta, quando os atletas ainda apresentam níveis inferiores de fadiga

Conexões: revista da Faculdade de Educação Física da UNICAMP, Campinas, v. 8, n. 2, p.174-186, maio/ago. 2010. ISSN: 1983-9030. 
neuromuscular; no entanto, em algumas situações, as pontuações acontecem com maior intensidade no final do combate, denotando que os atletas mais bem condicionados tendem a deixar a luta correr para, então, esforçarem-se após o quinto minuto de luta (DEL VECCHIO et al., 2007).

\section{FORÇA MUSCULAR APLICADA AO JIU-JITSU}

Força é uma grandeza física expressa pela massa versus aceleração, no treinamento físico é mais correto o uso da expressão força muscular (BARBANTI, 2001) que pode ser entendida como a força máxima que pode ser gerada por um músculo ou por um grupo muscular (POWERS; HOWLEY, 2000).

Em praticantes de jiu-jitsu é de fundamental importância um bom desenvolvimento da força, principalmente a de membros superiores através de contrações isométricas por sua utilização, devido à técnica ser em geral de extremo contato e não proporcionar espaços para movimentos dinâmicos (MOREIRA et al., 2003). Del Vecchio et al. (2007) indicam que os atletas de Brazilian Jiu-Jitsu necessitam de alta força isométrica.

Franchini et al., (2003) em pesquisa realizada com atletas de Brazilian Jiu-Jitsu ( $n=22)$ em simulações de luta de duração de $5 \mathrm{~min}$, relatam que os atletas desta modalidade não possuem elevada força de preensão manual, 54,2 \pm 6,7 kgf na mão direita e 51,4 \pm 6,1 kgf na mão esquerda, porém, conseguem mantê-la sem grandes alterações no decorrer de uma luta de 5 minutos, $84,4 \pm 7,7 \%$ da força de preensão máxima no quinto minuto de luta. Entre tanto na coleta dos dados havia interrupções a cada minuto, fato este que pode influenciar na manutenção da força, tendo os atletas pausas breve, nas quais podiam recuperar-se.

Em mesmo teste de dinamometria de preensão manual judocas da seleção brasileira apresentaram 49,5 \pm 12,8 kgf na mão direita e 47,2 \pm 12, 4 kgf na mão esquerda (FRANCHINI et al., 1997), enquanto judocas belgas de alto nível apresentaram 64, $9 \pm$ 8,9 kgf na mão direita e 59,7 \pm 8,8 kgf na mão esquerda em teste de preensão manual (CLAESSENS et al., 1984). Porém, deve-se destacar que a força de preensão manual tende a aumentar à proporção ao aumento da estatura, e com o aumento da massa corporal (FRANCHINI; DEL VECCHIO, 2001), e as circunferências de braço relaxado e contraído e de antebraço influenciam positivamente os resultados de preensão manual, apesar de essa correlação ser moderada (MOREIRA et al., 2003).

O treinamento de força é uma pratica normal no esporte de alto rendimento, incluindo as lutas (HÄKKINEN; KRAEMER, 2004). É indicado que após a preparação geral a força seja desenvolvida em

Conexões: revista da Faculdade de Educação Física da UNICAMP, Campinas, v. 8, n. 2, p.174-186, maio/ago. 2010. 
situações envolvendo técnicas especificas de luta (BLAIS; TRILLES, 2006). Porém, o treinamento deve sempre respeitar o âmbito da exigência especifica do esporte (BARBANTI, 2001).

No caso das lutas, os lutadores têm de apresentar potência vencendo grande resistência (gravidade, defesa do adversário). Desta forma, é indicado que o treinamento da potência seja realizado contra resistências significantes (HÄKKINEN; KRAEMER, 2004). Para desenvolvimento da força explosiva em lutas é indicado cargas entre 70 a $90 \%$ da força máxima. A magnitude das cargas deve ser maior se a necessidade é o desenvolvimento da força potente, e menor se a intenção é a força de saída (PLATONOV, 2004).

A força isométrica é muito solicitada nas lutas de combate de domínio, em especial para manter uma boa pegada, imobilizações e finalizações (DEL VECCHIO et al., 2007; FRANCHINI; DEL VECCHIO, 2008). O treinamento isométrico refere-se à ação muscular durante a qual não ocorre nenhuma alteração no comprimento total do músculo, este se tem mostrado efetivo para aumentar a força isométrica, e tem sido indicado que o treinamento seja diário para maximizar os resultados, embora, três sessões semanais em dias alternados resultem em aumento da força isométrica (FLECK; KRAEMER, 2006).

\section{POTÊNCIA E CAPACIDADE AERÓBIA APLICADAS AO JIU-JITSU}

Potência aeróbia máxima, $\mathrm{VO}_{2 m a ́ x}$, é uma medida reproduzível da capacidade do sistema cardiovascular de liberar sangue a uma grande massa muscular envolvida num trabalho dinâmico (POWERS; HOWLEY, 2000). Capacidade aeróbia é a capacidade máxima de transportar e utilizar oxigênio. Considera-se o poder aeróbio um importante indicador de aptidão física cardiovascular (LATIN, 1997).

A capacidade aeróbia é indicada pelo limiar anaeróbio, momento onde passa a prevalecer o metabolismo anaeróbio (POWERS; HOWLEY, 2000). Conhecer a zona de transição aeróbia/anaeróbia dos atletas de lutas se faz importante para controle do trabalho através da FC, individualizando o número de combates realizado por cada atleta na sessão de treino, pois uma sustentação por longo período do trabalho acima desta zona pode influenciar negativamente nos aspectos neuromusculares que exige a modalidade (RODRIGUEZ et al., 2007).

Acredita-se que valores elevados da potência e da capacidade aeróbia proporcionem ao atleta manter uma intensidade elevada durante toda a luta, retardando o acúmulo de lactato, e proporcione maior recuperação entre as lutas (CASTARLENAS; SOLÉ, 1997).

Conexões: revista da Faculdade de Educação Física da UNICAMP, Campinas, v. 8, n. 2, p.174-186, maio/ago. 2010. ISSN: 1983-9030. 
Atletas de judô norte-americanos de elite apresentaram $\mathrm{VO}_{2 m a ́ x}$ entre 53,2 \pm 1,4 $\mathrm{ml} \mathrm{kg} / \mathrm{min}$ (CALLISTER et al., 1990). Judocas da seleção canadense apresentaram valores de 59,2 $\pm 5,2 \mathrm{~m} / \mathrm{kg} / \mathrm{min}$ (THOMAS et al., 1989). Não existe exigência de valores acima de $65 \mathrm{mlkg} / \mathrm{min}$, nem indicações de que o $\mathrm{VO}_{2 \text { máx }}$ acima desse valor possa trazer vantagens, uma vez que o treinamento concorrente pode diminuir a capacidade do desenvolvimento de força e potência. Em atletas com mais de $90 \mathrm{~kg}$ tem sido comum encontrar valores de $\mathrm{VO}_{2 \text { máx }}$ abaixo de $50 \mathrm{mlkg} / \mathrm{min}$ (FRANCHINI, 2001).

$\mathrm{O}$ treinamento para melhorar o $\mathrm{VO}_{2 \text { máx }}$ é importante para atletas que competem em provas de média duração (2-3 minutos a 10-15 minutos) (DENADAI, 2000). Atividades com intensidade sub-máxima, tanto intervaladas quanto contínuas, não são capazes de aumentar o $\mathrm{VO}_{2 m a ́ x}$ em atletas que já possuem uma elevada potência aeróbia. Desta maneira tem sido comum a utilização do método intervalado, o qual tem se mostrado eficiente para o aumento da performance competitiva. Este método produz intensidade igual ou superior à encontrada na competição (BARBANTI et al., 2004).

Em atletas altamente treinados se faz indispensável ao menos uma ou duas sessões de treinamento intervalado de alta intensidade, acima do $\mathrm{VO}_{2 m a ́ x}$, para que ocorra melhora do $\mathrm{VO}_{2 m a ́ x}$. Porém, a intensidade e volume devem respeitar a individualidade do atleta para evitar o overtraining (DENADAI, 2000). Exercícios de tiros e alto intervalo, 90 segundos a 3 minutos, tendem a não aumentar o $\mathrm{VO}_{2}$ de Pico, diferente do treinamento com menor tempo de intervalo, entre 20segundos e 1 minuto (FLECK; KRAEMER, 2006).

No treinamento aeróbio é mais indicado que seja realizado se valendo de situações especificas de luta (IGUMA, 1991). Franchini; Del Vecchio (2008) indicam que exercícios gerais como corrida e ciclismo são mais indicados no período inicial da preparação quando o atleta apresentar baixa potência e/ou capacidade aeróbia; em recuperação de lesões; ou em período transitório. Entre tanto, o $\mathrm{VO}_{2 m a ́ x}$ não é o único determinante em eventos de resistência, tendo o limiar de lactato, que é ponto onde o acúmulo de LAC é maior que os níveis de remoção durante exercícios com incrementos constantes de intensidade; e a fração de $\mathrm{VO}_{2 \text { máx }}$ tem grande importância neste processo. A fração do $\mathrm{VO}_{2 \text { máx }}$ reflete a fração empregada na realização de uma atividade, sendo que atletas com o mesmo $\mathrm{VO}_{2 m a ́ x}$ podem realizar atividades iguais utilizando frações distintas do $\mathrm{VO}_{2 \text { máx }}$. Esta distinção nas frações se deve à vantagem mecânica adquirida com aprimoramento técnico (BARBANTI et al., 2004; DENADAI, 2000).

O treinamento aeróbio pode ser controlado pela FC, percepção subjetiva de esforço ou [LAC]. Neste ultimo método se deve respeitar 3-4 $\mathrm{mMol}^{-1}$ para aumentar a capacidade aeróbia, e 5-6 mMol.1 ${ }^{-1}$ para

Conexões: revista da Faculdade de Educação Física da UNICAMP, Campinas, v. 8, n. 2, p.174-186, maio/ago. 2010. 
aumento da capacidade aeróbia (FRANCHINI; DEL VECCHIO, 2008).

\section{POTÊNCIA ANAERÓBIA APLICADA AO JIU-JITSU}

Potência anaeróbia representa uma característica local de um músculo existir independentemente do suprimento de sangue e oxigênio (FOSS; KETEYIAN, 2000). Esta por sua vez é determinada pela capacidade de acelerar, pela magnitude da velocidade máxima e pela capacidade de suportar a fadiga (FLECK; KRAEMER, 2006). Para sua avaliação é essencial que o teste empregado envolva os grupos musculares envolvidos no esporte e inclua as vias energéticas utilizadas no evento. Os testes podem ser de dois tipos, teste de duração ultracurta o qual visa avaliar a capacidade máxima do sistema ATP-CP; e teste de duração curta, que tende avaliar a capacidade máxima da glicólise anaeróbia (POWERS; HOWLEY, 2000). Atletas de Brazilian Jiu-Jitsu (n=7) em teste de Wingate apresentaram excelente potência anaeróbia, tanto de pico quanto média (DEL VECCHIO et al., 2007).

O treinamento anaeróbio para o judô tem sido direcionado para (1) induzir por meio de atividades especificas da modalidade respostas na [LAC] similares às observadas nas competições, assim como simulações com períodos de esforço e pausa semelhantes às encontradas em competições; (2) adotar valores supramáximos de consumo de oxigênio, assumindo uma relação linear entre a carga de trabalho e a solicitação fisiológica, normalmente esse treinamento é realizado em situações não especificas, mas que podem ser adaptadas ao treinamento especifico da modalidade; (3) series de exercícios onde o executante realize o maior esforço possível em período fixo com intervalos para a recuperação que considere a ressíntese de alguns substratos energéticos ou a remoção de metabólitos das vias anaeróbias; e (4) execução de exercícios intermitentes com intensidade, duração de esforço e pausa similares ao que ocorre durante a competição (FRANCHINI; DEL VECCHIO, 2008).

Quanto ao seu desenvolvimento em meio não especifico, tiros de corrida de poucos segundos requerem maior produção de potencia do que tiros de velocidade de longa duração, 1 a 2 minutos (FLECK; KRAEMER, 2006; WILMORE; COSTILL, 2001). Caso as pausas não sejam suficientes para a regeneração das vias energéticas, o trabalho ganha caráter aeróbio apesar da intermitência (DENADAI, 2000).

\section{VELOCIDADE APLICADA AO JIU-JITSU}

Velocidade motora pode ser entendida como uma qualidade física no movimento humano, a capacidade de executar num espaço de tempo mínimo, ações motoras sob exigências dadas (BARBANTI, 2001) ou

Conexões: revista da Faculdade de Educação Física da UNICAMP, Campinas, v. 8, n. 2, p.174-186, maio/ago. 2010. 
como capacidade, com base na mobilidade dos processos do sistema nervo-músculo e da capacidade de desenvolvimento da força muscular, de completar ações motoras sob determinadas condições, no menor tempo (WEINECK, 1991).

A velocidade pode ser dividida em (1) velocidade de reação, a qual ainda pode ser subdividida em (a) simples, que representa uma resposta estereotipada a um estímulo desencadeador estereotipado, e (b) complexa onde contém geralmente uma reação seletiva a sinais variados; (2) velocidade acíclica, a qual contém as ações ou movimentos únicos motores; e (3) velocidade cíclica, que consiste numa seqüência de ações motoras, ritmicamente repetida, independente do fato de se tratar de movimentos das extremidades superiores ou inferiores, assim como do tronco (WEINECK, 2003).

A velocidade de reação que é o intervalo entre o momento de apresentação de sinal externo e o inicio da resposta muscular apropriada, é essencial para lutadores, uma vez que para realizar o ataque no decorrer da luta o atleta tem de estar atento às ações do adversário para que possa manter o ataque, contra golpear ou esquivar-se. Isto depende do grau de atenção e da velocidade de reação (LIMA et al., 2004). Porém, Platonov (2004) aponta que em esportes de lutas os atletas não têm tempo para reagir à ação propriamente dita, desta forma os atletas utilizam-se de processos antecipatórios a sinais expressos que levariam a determinada ação, assim eles antecipam a defesa antes mesmo do ataque ser realizado.

A velocidade depende de fatores como força básica, a qual expressa sob a forma de força rápida, importante na aceleração do corpo em movimentos cíclicos; coordenação, sendo que quanto mais coordenado o movimento mais rapidamente a excitação e inibição serão alternados; viscosidade das fibras musculares, relação de alavancas extremidades-tronco, sendo estes fatores constitucionais, não sendo modificados com o treinamento; e elasticidade muscular, importante para a velocidade de contração, sendo que uma musculatura antagônica limitada refreia as ações motoras em sua fase final, diminuindo a velocidade do movimento, e para uma elevada velocidade de contração se faz necessário um ótimo relaxamento da musculatura não atuante no movimento, o que só se pode atingir com ideal elasticidade muscular (BARBANTI, 2001).

A velocidade de contração depende do teor de ATP na musculatura e de sua ressíntese que se dá quase exclusivamente pelo CP e pelo metabolismo glícolítico. Ainda como fator determinante pode relatar-se a proporção entre fibras lentas e rápidas, uma vez que a velocidade da transmissão de estímulo é maior em fibras rápidas, as quais possuem mais $\mathrm{CP}$ e realizam atividade enzimática fosfática maior (BARBANTI, 2001).

Conexões: revista da Faculdade de Educação Física da UNICAMP, Campinas, v. 8, n. 2, p.174-186, maio/ago. 2010. 
Iguma (1991) recomenda para o judô que o trabalho para melhora da velocidade seja realizado com gesto técnico especifico, na maior velocidade possível. Entre tanto o número de repetições deve ser baixo, em torno de 5 execuções, o intervalo deve ser recuperador, em torno de 2 a 5 minutos e o volume deve ser composto de 6 a 10 séries, e a frequiência por volta de três vezes semanais. Contudo, o autor relata que a sobrecarga pode ser aumentada com um aumento no numero de repetições, e se faz importante que o treino seja realizado sem fadiga prévia.

\section{FLEXIBILIDADE APLICADA AO JIU-JITSU}

O American College of Sports Medicine (ACSM, 2003) define flexibilidade como capacidade de movimentar uma articulação por meio de sua amplitude de movimento completo; e é importante tanto no desempenho atlético como em atividades diárias.

Cada articulação do corpo permite uma amplitude de movimento específica. Ocorre falta de flexibilidade individual se a pessoa for incapaz de produzir a amplitude de movimento a que cada articulação é destinada (BLANKE, 1997). Entre tanto, um grau elevado de flexibilidade de todas as articulações pode não ser desejável em alguns esportes, sendo a flexibilidade excessiva em geral um indicativo de propensão a lesões no esporte de contato. O aumento da massa muscular pode diminuir a flexibilidade, mas reduz também as chances de lesões por aumentar a estabilidade (POWERS; HOWLEY, 2000).

As artes marciais exigem hiperextensibilidade em determinadas articulações, específicas de cada esporte. O Brazilian Jiu-Jitsu exige um bom nível de amplitude de tronco para execução de seus movimentos específicos. O treinamento da flexibilidade permite uma maior amplitude de movimento, e permite maior velocidade na execução dos movimentos, devido ao fato que uma maior distância existente entre a origem e inserção muscular proporciona maior campo de ação para o desenvolvimento da velocidade, ainda uma boa flexibilidade facilita o aprendizado e execução dos gestos técnicos específicos de cada modalidade de luta. De forma geral é vantajoso lutadores apresentarem boa flexibilidade em todas as articulações do corpo (CASTAÑEDA, 2004). O desenvolvimento da flexibilidade parece ser importante em alguns aspectos como a luta no solo, contudo pode não ser um determinante de sucesso esportivo (ROSA, 2006).

Não existe um único teste que possa ser usado para avaliar a flexibilidade corporal total (ACSM, 2003). No teste de Banco de Wells e Dillon, Del Vecchio et al., (2007) apontam que atletas de Jiu-Jitsu (n=7) apresentaram valores de 42,9 $\pm 3,0 \mathrm{~cm}$, concluindo que os sujeitos apresentaram bons resultados nesse 
teste, ainda mais quando comparado o resultado com valores obtidos em pesquisa com a seleção brasileira universitária de Judô (n=5) que apresentaram valores de 36,2 \pm 4,7 cm. Souza et al., (2005) em pesquisa com atletas de Jiu-Jitsu $(n=46)$ revelam haver uma forte associação entre o tempo de prática e a flexibilidade tóraco-lombar e de quadril, sugerindo que essa flexibilidade aumenta sob influência do treinamento, o que pode ser um importante componente no desenvolvimento e no desempenho de atletas de Jiu-Jitsu, uma vez que as articulações tóraco-lombar e do quadril são constantemente solicitadas. No flexiteste os atletas apresentaram valores relativamente altos, porém com baixa correlação entre ambos.

Quanto ao desenvolvimento da flexibilidade, existem diversos métodos para desenvolver flexibilidade com o objetivo de conquistar amplitudes ótimas de movimento. Inicialmente, é preciso identificar algumas características pessoais, como condições de adaptação a um dos métodos, a facilidade em realizar os exercícios e sua idade, e não apenas escolher o método mais rápido para desenvolver flexibilidade (ACHOUR JUNIOR, 2006). O treinamento de flexibilidade deve considerar as articulações específicas que são exigidas na modalidade, e torna-se importante a execução dos movimentos empregando grande velocidade para assemelhar-se com a ação na luta. Pode-se dividir este treinamento em duas fases, onde a primeira fase objetiva-se o aumento da amplitude de movimento. Nesta fase devese realizar trabalhos diários; e na segunda fase objetiva-se a manutenção da amplitude de movimento, na qual realiza-se trabalhos de 3 a 4 vezes por semana (CASTAÑEDA, 2004). Fator Importante durante o treinamento é o principio da continuidade, pois a inatividade fará com que a flexibilidade volte aos níveis iniciais. (BARBANTI, 2001).

\section{CONSIDERAÇÕES FINAIS}

Ao analisar o treinamento desportivo aplicado ao Brazilian Jiu-jitsu se pode perceber que ainda existem diversas lacunas a serem preenchidas, visto a evolução do esporte. Com base em dados disponíveis da modalidade, e com bases em dados de modalidades semelhantes, como luta olímpica e judô, pode-se relatar que a principal base a ser seguida é a definição de quais fatores são determinantes e quais fatores são limitantes do desempenho em lutas de Brazilian jiu-jitsu.

A partir disto, deve-se conduzir o treinamento com a maior especificidade possível, evitando a inclusão de exercícios gerais e que não se enquadre nas demandas energéticas da modalidade. Contudo, devem ser estimulados mais estudos acerca do Brazilian Jiu-jitsu, visto que ainda existem muitas questões a serem investigadas.

Conexões: revista da Faculdade de Educação Física da UNICAMP, Campinas, v. 8, n. 2, p.174-186, maio/ago. 2010. ISSN: 1983-9030. 


\section{REFERÊNCIAS}

ACHOUR JUNIOR, A. Exercícios de alongamento: anatomia e fisiologia. 2. ed. Barueri: Manole, 2006. AMERICAN COLLEGE OF SPORTS MEDICINE. Diretrizes do ACSM para os testes de esforço e sua prescrição. 6. ed. Rio de Janeiro: Guanabara Koogan, 2003.

ARTIOLI, G. G.; FRANCHINI, E.; LANCHA JÚNIOR, A. H. Perda de peso em esportes de combate de domínio: revisão e recomendações aplicadas. Rev Bras Cineantr Des Hum, v. 8. 2006. p. 92-101.

ASSIS, M. M. V.; GOMES, M. I.; CARVALHO, E. M. S. Avaliação isocinética de quadríceps e ísquiostibiais nos atletas de jiu-jitsu. Rev Bras Prom Saúde, p. 85-89, 2005.

BLANKE, D. Flexibilidade. In: MELLION, M. B. et al. Segredos em medicina desportiva: respostas necessárias ao dia-dia em centros de treinamento, na clínica, em exames orais e escritos. Porto Alegre: Artes Médicas, 1997.

BARBANTI, V. J.; TRICOLI, V.; URGRINOWITSCH, C. Relevância do conhecimento científico na pratica do treinamento físico. Rev Paul Educ Fis, v. 18, p. 101-109, 2004.

BARBANTI, V. J. Treinamento físico: bases científicas. São Paulo: CLR Baleiro, 2001.

BLAIS, L.; TRILLES, F. The progress achieved by judokas after strength training with a judo-specific machine. J Sports Sci Med, v. 5, 2006.

CALLSITER, R.; et al. Physiological and performance responses to overtraining in elite judo athletes. Med Sci Sports Exercise, v. 22, n. 6, p.S816-824, 1990.

CASTAÑEDA, P. E. G. Importancia del desarrollo óptimo de la flexibilidade en las artes marciales. EFDeportes: revista digital, Buenos Aires, n. 69, 2004.

CASTARLENAS, J. L.; SOLÉ, J. El entrenamiento de la resistencia en los deportes de lucha con agarre: una propuesta integradora. Apunts: Educ Fis Dep, v.1, n.47, p.81-6, 1997.

CLAESSENS, A. et al. Somatotype and body structure of world top judoists. J Sports Med Phys Fitness, v. 27, n. 1, p. 105-13, 1987.

DENADAI, B. S. Intensidade e tempo de exaustão a 100\%: implicações para o treinamento e a performance. Rev Educ Fis, v. 1, n. 124, p. 23-30, 2000.

DEL VECCHIO, F. B. et al. Análise morfo-funcional de praticantes de brazilian jiu-jitsu e estudo da temporalidade e da quantificação das ações motoras na modalidade. Mov Percepc, v. 7, n. 10, p. 263-281, 2007.

DRIGO, A. J. et al. Demanda metabólica em lutas de projeção e de solo no judô: estudo pelo lactato sangüíneo. Motriz, v.2, n 2., p. 80-86, 1996. 
FLECK, S. J.; KRAEMER, W. J. Fundamentos do treinamento de força muscular. 3. ed. Porto Alegre: Artmed, 2006.

FORTEZA, A. Entrenamiento deportivo: alta metodologia. Cuba: Komekt, 1999.

FOSS, M. L.; KETEYAN, S. J. Bases fisiológicas do exercício e do esporte. 6. ed. Rio de Janeiro: Guanabara Koogan, 2000.

FRANCHINI, E. Judô: desempenho competitivo. Barueri: Ed. Manole, 2001.

.; DEL VECCHIO, F. B. Preparação física para atletas de Judô. São Paulo: Phorte, 2008.

FRANCHINI, E.; TAKITO, M. Y.; PEREIRA, J. N. D. C. Freqüência cardíaca e força de preensão manual durante a luta de jiu-jitsu. EFDeportes: revista digital, Buenos Aires, n. 65, out. 2003. Disponível em: 〈http://efdeportes.com>. Acesso em: 15 jul. 2009.

FRANCHINI, E. et al. Composição corporal, somatotipo e força isométrica em atletas da seleção brasileira universitária de judô. Âmbito Med Esp, v.3, n. 4, p. 21-29,1997.

Influência da aptidão aeróbia sobre o desempenho em uma tarefa anaeróbia láctica intermitente. Motriz, v.5, n. 1, p. 58-66, 1999.

GOMES, A. C. Treinamento desportivo: estrutura e periodização. Porto Alegre: Artmed, 2001.

HÄKKINEN, K.; KRAEMER, W. J. Treinamento de força para o esporte. Porto Alegre: Artmed, 2004.

IGUMA, E. H. Aspectos do treinamento total no Judô. Rev Educ Fis, v. 1, n. 119, p. 49-63, 1991.

LATIN, R. W. Construindo a capacidade aeróbia. In: MELLION, M. B. et al. Segredos em medicina desportiva: respostas necessárias ao dia-dia em centros de treinamento, na clínica, em exames orais e escritos. Porto Alegre: Artes Médicas, 1997.

LIMA, E. V. et al. Estudo da correlação entre a velocidade de reação motora e o lactato sangüíneo em diferentes tempo de luta no Judô. Rev Bras Med Esporte, v.10, n.5, p. 339-343, 2004.

LÔBO, I. L. B.; MORAES, L. C. C. A.; NASCIMENTO, E. Processo de validação da escala de comportamento do treinador-versão atleta (ECT-A). Rev Bras Educ Fis Esportes, v. 19, n. 3, p. 255-265, 2005 .

MOREIRA, S. R. et al. Correlação de variáveis antropométricas de membros superiores com a força de preensão manual em praticantes de jiu-jitsu. Motriz, v. 9, n.1, p. S147-148, 2003.

PLATONOV, V. N. Teoria geral do treinamento desportivo olímpico. 6. ed. Porto Alegre: Artmed, 2004. POWERS, S. K.; HOWLEY, E. T. Fisiologia do exercício: teoria e aplicação ao condicionamento e ao desempenho. 3. ed. Barueri: Manole, 2000.

RODRIGUEZ, L. S. et al. ¿ Por qué ES importante conocer la zona de transición aeróbica-anaeróbica en

Conexões: revista da Faculdade de Educação Física da UNICAMP, Campinas, v. 8, n. 2, p.174-186, maio/ago. 2010. 
el judo del competición? EFDeportes: revista digital, Buenos Aires, n. 104, 2007. Disponível em: <http://efdeportes.com>. Acesso em: 15 jul. 2009.

ROSA, R. R. Testes de controle no judô: proposta de avaliação da resistência especial do judoca. 2006. Dissertação (Mestrado) - Faculdade de Educação Física, Universidade Estadual de Campinas, Campinas, 2006.

SOUZA, I.; SILVA, V. S.; CAMÕES, J. C. Flexibilidade tóraco-lombar e de quadril em atletas de jiujitsu. Revista Digital EFDeportes, Buenos Aires, n. 82, mar. 2005. Disponível em: <http://efdeportes.com>. Acesso em: 15 jul. 2009.

THOMAS, S. G. et al. Physiological profiles of the Canadian National Judo Team. Can J Sport Sci, v. 14, n. 3, p.142-147, 1989.

TUBINO, M. J. G. Metodologia científica do treinamento desportivo. 3. ed. São Paulo: Ibrasa, 1984.

WEINECK, J. Biologia do esporte. São Paulo: Manole, 1991.

. Treinamento ideal. Barueri: Manole, 1999.

WILMORE, J. H.; COSTIL, D. L. Fisiologia do esporte do exercício. São Paulo: Manole, 2001.

\section{LEONARDO VIDAL ANDREATO}

Universidade Federal de São Paulo - UNIFESP, São Paulo-SP

\section{Referencia do artigo}

\section{ABNT}

ANDREATO, L. V. Bases para prescrição do treinamento desportivo aplicado ao brazilian jiu-jitsu. Conexões, v. 8, n. 2, p. 174-186, 2010.

\section{APA}

Andreato, L. V. (2010). Bases para prescrição do treinamento desportivo aplicado ao brazilian jiu-jitsu. Conexões, 8(2), 174-186.

\section{VANCOUVER}

Andreato LV, Bases para prescrição do treinamento desportivo aplicado ao brazilian jiu-jitsu. Conexões, 2010; 8(2), 174-186.

Recebido em: dez./2009

Aceito para publicação em: mar./2010

Conexões: revista da Faculdade de Educação Física da UNICAMP, Campinas, v. 8, n. 2, p.174-186, maio/ago. 2010. 